\title{
A morte materna no Brasil: razão e sensibilidade
}

\section{Maternal death in Brazil: sense and sensibility}

As estimativas globais sobre o declínio da mortalidade materna revelaram que os resultados atingidos não serão suficientes para alcançar, na maioria dos países, as Metas de Desenvolvimento do Milênio, de reduzir em 75\% a razão da mortalidade materna em 15 anos $^{1,2}$. Em 2010, havia sido alcançada a redução de $47 \%$, tomando como ano a razão de 1990, o que significa em números absolutos que aproximadamente 287.000 mortes maternas ocorreram em todo o mundo. Duas regiões se destacam: a África Subsaariana (56\%) e o Sul da Ásia (29\%), que respondem por $85 \%$ do número absoluto total (245.000 mortes maternas) em 2010. Apenas dois países são responsáveis por um terço das mortes maternas globais: a Índia, com $19 \%$ (56.000) e a Nigéria, com 14\% (40.000). A razão de morte materna em 2010 foi de 210 mortes maternas por 100.000 nascidos vivos, ou seja, quase a metade das 400 mortes maternas por 100.000 nascidos vivos em 1990. Nesta mesma publicação, devido ao método e ao fator de correção empregados, a razão para a América Latina e o Caribe é de 80, e a do Brasil é de 56 , dentro de um intervalo com um limite de confiança entre 36 e 85 com número absoluto de mortes de $1998^{3}$.

Controvérsias metodológicas e números absolutos a parte, todos concordam que a redução foi importante, mas insuficiente, e que será necessário aumentar a velocidade de declínio no tempo remanescente para alcançar a meta estipulada. Em setembro de 2010, o Secretário Geral da ONU lançou a "Estratégia Global de Saúde de Mulheres e Crianças", buscando mobilizar apoio por parte dos governos, organizações da sociedade civil e outros parceiros em resposta à necessidade de acelerar esforços para salvar a vida e melhorar a saúde das mulheres e recém-nascidos ${ }^{4}$. As Nações Unidas reconhecem que existe uma lacuna entre o investimento necessário e o que está atualmente previsto para a saúde das mulheres e crianças, e que para produzir mudanças previstas é necessário incrementar os recursos financeiros em cerca de U \$40 bilhões ao longo dos próximos cinco anos ${ }^{5}$.

Com os mesmos objetivos, em 2011, os Ministros da Saúde da região das Américas e Caribe presentes no $51^{\circ}$ Conselho Diretivo da Organização Pan-Americana de Saúde (OPAS) aprovaram, por unanimidade, o plano de Ação para acelerar a redução da mortalidade materna e morbidade materna grave 2012-2017 ${ }^{6}$. Este plano de ação está centrado na melhoria da resposta do setor da saúde, com quatro áreas estratégicas: 1) prevenção da gravidez indesejada e suas complicações, com solução aos problemas de acesso universal a contraceptivos de

Correspondêncio Suzanne Jacob Serruya Hospital de Clínicas Av. Itália s/n

CLAP - Piso 16 - 11.400 Montevidéu, Uruguai

08/10/2012

Aceito com modificações

$01 / 11 / 2012$
Organização Pan-Americana de Saúde da Organização Mundial da Saúde - OPAS/OMS; Centro Latino-Americano de Perinatologia/Saúde da Mulher e Reprodutiva - CLAP/SMR

Conflitos de interesse: não há

Declaração de isenção de responsabilidade: A autora deste comentário editorial é o responsável pelo seu conteúdo. As visões expressas neste documento não refletem, necessariamente, as posições oficiais da Organização Pan-americana de Saúde da Organização Mundial da Saúde. 
qualidade e redução do aborto inseguro; 2) acesso universal a serviços para parto, ou a preços acessíveis, garantindo que os países eliminem as barreiras econômicas para o acesso aos serviços, aumento da qualidade e priorização das populações vulneráveis; 3) qualificação de recursos humanos, promovendo o aumento do número e da qualidade dos mesmos, melhorando a capacidade de resposta de emergência e tratamento de complicações obstétricas; 4) fortalecimento dos sistemas de informações estratégicas para ação e accountability, com melhoria da vigilância materna e perinatal e do estabelecimento dos comitês de análise de morte materna.

No Brasil, desde o Programa de Atenção Integral à Saúde da Mulher, iniciativas regulatórias e normativas vêm sendo estabelecidas no campo da saúde materna. Recentemente, o programa Rede Cegonha foi acrescentado a estas estratégias de saúde materna e infantil. A saúde materna foi declarada prioritária pelo governo federal, e criou-se um programa com fundos muito superiores aos destinados programaticamente: $\mathrm{R}$ \$9,397 bilhões. Compreendendo que só é possível realizar mudanças se as condições objetivas estiverem garantidas, o estabelecimento de financiamento é muito significativo do ponto de vista das políticas a serem implementadas ${ }^{7}$.

\section{As razões e suas evidências}

Múltiplos fatores podem explicar o declínio global, regional e nacional da mortalidade materna entre 1990 e 2010. Entre estes, destacam-se o fortalecimento do sistema de saúde, o acesso aos serviços e a melhoria do nível educacional das mulheres. No Relatório Mundial da Saúde, publicado pela OMS em $2010^{8}$, o tema de financiamento da cobertura universal foi discutido como um dos elementos fundamentais para o avanço dos sistemas de saúde. Este direito, conquistado no Brasil há mais de duas décadas, ainda não é realidade em todos os países da região. Numa estimativa conservadora, cerca de 20 a 40\% dos recursos da saúde são desperdiçados. De maneira simbólica para a discussão em tela, o relatório global tomou como exemplo a epidemia de cesáreas. O número de cesáreas varia muito entre os países, com os mais ricos e os em transição registrando um recurso excessivo a este procedimento. Já os países economicamente mais frágeis, principalmente da África, não conseguem responder à demanda. Os dados sobre cesáreas em 2007 mostraram que de 137 países, em 54 esta via representava menos de $10 \%$ de todos os nascimentos, e em 69 países, a percentagem era superior a $15 \%$. Apenas 14 países reportavam taxas dentro dos níveis recomendados, de 10 a 15\%. Uma análise em nível nacional, baseada no método WHO-CHOICE (Choosing Interventions that Are Cost Effective), revelou que o custo global do excesso de cirurgias é superior a US\$ 2 bilhões anualmente. Em 2008, o total de cesáreas desnecessárias superou as realmente necessárias. Devido à concentração esmagadora de cirurgias desnecessárias em países com elevados níveis de rendimento (e, por isso, níveis de preços mais elevados), o custo global do excesso de cesáreas em 2008 poderia ter potencialmente financiado, por mais de seis vezes, os procedimentos realmente necessários nos países mais pobres.

O trabalho de Souza et al. ${ }^{9}$ publicado neste fascículo cita o risco elevado relativo de mortes maternas para cesárea em relação ao parto vaginal, assim como sua associação com complicações como hemorragias, infecções, embolia pulmonar e acidentes anestésicos, mas não discute nos casos apresentados para esta causa a relação via de parto e complicação. Seria importante conhecer a via de parto dos casos relacionados para entender a contribuição da intervenção cirúrgica versus causa da mortalidade. Talvez seja muito complicado responder se eram cesáreas cuja indicação era questionável ou não, mas seria importante de conhecer a via de parto para os casos fatais.

Entre os outros fatores relacionados com a diminuição das mortes maternas está a proporção crescente de partos assistidos por profissional de saúde qualificado nos países em desenvolvimento: de 55\%, em 1990, a 65\%, em 2009. Na região das Américas, a OPAS informa que a taxa de partos atendidos por profissional qualificado é de $95,5 \%$, sendo que no Brasil chega-se a 99\%. Globalmente, a proporção de mulheres que foram atendidas por pessoal qualificado pelo menos uma vez durante a gravidez aumentou de $64 \%$ para $81 \%$, enquanto que a proporção de mulheres com idade entre 15-49 anos que utiliza qualquer método de contracepção também aumentou de $52 \%$ para $61 \%$. No país, o número de mulheres que tem quatro consultas de pré-natal é de $99 \%$, e na região das Américas, 94\%. Já a taxa de uso de métodos contraceptivos na região é de $64 \%$ e, no Brasil, de $78 \% 2$.

Estes indicadores guardam estreita relação com a morte materna. Entretanto, focando a análise no Brasil, é necessário primeiro reconhecer que o bom resultado no indicador nacional, se desagregado, revela iniquidades importantes. No Brasil, por exemplo, o estado do Maranhão apresenta apenas 23,6\% das mães de nascidos vivos com sete ou mais consultas de pré-natal, enquanto no Paraná a porcentagem é de $80,4^{3}$.

Analisando dados desagregados do estado paranaense, Soares, em $2007^{10}$, avaliou as desigualdades sociais e em saúde reprodutiva das mulheres em um estudo ecológico, cujas unidades de análise foram os municípios paranaenses agregados em áreas homogêneas segundo indicadores socioeconômicos (porcentagem de população rural, porcentagem de famílias pobres, renda municipal per capita). O estudo mostrou que são importantes as diferenças nos indicadores de saúde reprodutiva 
das mulheres entre os municípios, tanto na tipologia socioeconômica como na inserção social das mulheres, para mais de $40 \%$ da população total do Estado, cerca de 1,4 milhões de pessoas ${ }^{10}$. Usando as variáveis do Sistema de Informações sobre Nascidos Vivos (SINASC), Predebone et al. ${ }^{11}$ encontraram diferenças significativas na Região Metropolitana de Maringá em resultados maternos e perinatais: mãe adolescente, baixa escolaridade, raça/cor preta e parda, número insuficiente de consultas de pré-natal, parto cesáreo, prematuridade, baixo peso ao nascer e Apgar $<8$ no quinto minuto ${ }^{11}$.

$\mathrm{Na}$ discussão da mortalidade materna no Brasil e em seus estados, o reconhecimento dos determinantes sociais é fundamental porque, como demonstram estes dois estudos, as iniquidades são marcantes e podem explicar parte importante do problema. No artigo publicado neste fascículo" as variáveis escolhidas foram as causas dos óbitos, a transferência hospitalar e a evitabilidade, sem incluir os determinantes sociais que são relevantes para analisar as causas da mortalidade, ampliar a análise e proposições de intervenções e recomendações. Em muitos lugares, esta discussão ampliada tem permitido a proposição das intervenções necessárias para diminuir a morte evitável de mulheres.

$\mathrm{Na}$ mesma linha de análise, Koch et al. ${ }^{12}$ analisaram a série histórica dos resultados relativos à morte materna no Chile entre 1957 e $2007^{12}$. O país tem a menor razão de morte materna na América Latina e a segunda menor nas Américas, depois do Canadá, e apresentou uma tendência decrescente contínua até 2003. Para os autores, um elemento fundamental foi a implementação da política de educação, em 1965, com estreita relação com a saúde materna e significativo impacto sobre a tendência da mortalidade. Em relação à atenção por profissional de saúde e acesso a serviços de saúde, o percentual de partos assistidos por um profissional habilitado aumentou de 60,8\%, em 1957, para mais de 90\%, em 1980. Desde 1999, mais de $99 \%$ de todos os partos ocorrem em hospitais ou maternidades.

A Organização Mundial da Saúde (OMS) realizou, em 2005-2006, a Pesquisa Global sobre Saúde Materna e Perinatal em 373 instituições de 24 países da África, Ásia e América Latina, incluindo 287.035 mulheres ${ }^{13}$. Entre os resultados publicados estão os achados relativos à relação entre educação materna e mortalidade materna, a associação entre idade materna, estado civil, paridade, capacidade institucional e nível de investimento do estado na área da saúde com essas relações. Os principais resultados apontam que, nos modelos ajustados, as mulheres sem instrução tinham 2,7 vezes mais risco de morte materna, e as que tinham entre um e seis anos de escolaridade tiveram o dobro do risco de morte comparadas com as mulheres com mais de 12 anos de educação. Mesmo para as mulheres que tiveram parto hospitalar, a mortalidade foi mais elevada entre aquelas com níveis mais baixos de formação, e isto não pode ser explicado pelo nível de serviços disponíveis nas instituições em que se deu o parto. Ajustando também as variáveis de idade, estado civil, paridade e nível de investimento em serviços de saúde, não foi possível explicar a influência da educação sobre a mortalidade materna. Ou seja, mesmo para as mulheres capazes de ter acesso à assistência hospitalar durante o parto, os impactos negativos dos determinantes mais amplos da saúde, especificamente o nível educacional, permanecem associados à maior taxa de mortalidade. Para os autores, as mulheres com maior nível educacional são menos propensas a aceitar as explicações tradicionais e têm mais autonomia para tomar decisões importantes relacionadas ao comportamento reprodutivo ou à melhora da nutrição. São mais seguras para fazer perguntas sobre seus problemas de saúde e apresentam mais predisposição de serem escutadas por profissionais de saúde.

Com o objetivo de entender quais impactos, fatores e programas são efetivos para a melhoria da saúde materna, Nyamtema et al. ${ }^{14}$ realizaram uma revisão sistemática para explorar as evidências disponíveis, a integração de intervenções em saúde materna e os fatores que influenciam a implementação de estratégias em países com recursos limitados. Nos 54 programas analisados, os que integravam múltiplas intervenções eram mais propensos a terem impactos positivos sobre os resultados maternos. Os treinamentos em cuidados obstétricos de emergência (COE), disponibilização (placement) de prestadores de cuidados, reforma na infraestrutura das unidades de saúde existentes e melhoria do abastecimento de medicamentos, materiais de consumo e equipamentos para cuidados obstétricos foram as mais frequentes intervenções integradas em $52 \%$ a $65 \%$ de todos os 54 programas analisados. A redução estatisticamente significativa da razão de mortalidade materna e taxa de letalidade foi relatada em 55 e $40 \%$ dos programas, respectivamente. Nascimentos em ambiente hospitalar e taxas de cesarianas aumentaram significativamente, de 71 a $75 \%$, nos programas que utilizavam esses indicadores.

\section{Sensibilidade: toda mulher, toda criança}

Souza et al. ${ }^{9}$ concluíram que os dois grupos de causas não são muitos diferentes entre si nos dois triênios estudados, para os hospitais de referência de gestação de alto risco e hospitais de baixo risco. Este é um resultado esperado, mas os autores também verificaram que nos dois períodos a taxa de letalidade materna (TLM) total nos hospitais de referência para gestação de alto risco foi cerca de duas vezes superior às taxas dos hospitais de referência para o baixo risco. Espera-se que os casos referidos à maternidade de alto risco sejam mais complexos em termos de manejo clínico e obstétrico, mas 
seria muito interessante explorar, entre as práticas estabelecidas, esta diferença na letalidade para pelo menos uma causa. Em particular, causas em que a letalidade no hospital de alto risco foi muito superior ao de baixo risco, como na causa de infecção urinária, 13,7 versus 1,7, respectivamente. Outro exemplo é a infecção puerperal, três vezes superior nos hospitais de alto risco, que é associada às elevadas taxas de cesárea (dado não citado no artigo).

Nos hospitais de referência para gestação de baixo risco as complicações anestésicas, hemorragias e embolias são mais frequentes e estão relacionadas a duas práticas bem conhecidas: cesáreas desnecessárias e um único profissional realizando a cesárea e a anestesia. Na falta da equipe mínima necessária para o atendimento obstétrico, a mulher deveria ser encaminhada, principalmente se a cesárea é eletiva. Rudge et al. ${ }^{15}$ realizaram um estudo analisando os resultados do sistema de referência obstétrica em uma região de São Paulo. Entre os principais resultados desta estratégia, apontam: a diminuição da taxa de cesárea de 46,5 para 23,4\% no nível II, de baixo risco, do hospital, e a manutenção da taxa do nível III, considerado de alto risco. Houve uma mudança nas causas de mortalidade materna, de causas diretas para indiretas, com melhores resultados perinatais, ainda que a taxa de letalidade na referência para casos complexos fosse muito mais alta que a de baixo risco.

A discussão apresentada na análise de aborto resume com precisão as falhas relativas à atenção profissional e institucional, destacando a inadequação das condutas profissionais, o tratamento das emergências obstétricas e as demoras na resolução de complicações decorrentes do aborto. Este quadro tão comum mostra que há um enfrentamento ético destes casos, com a soma de dois elementos subjetivos: a culpabilização da mulher e a não priorização da atenção. Em quase todas as análises desta importante causa de mortalidade materna, o atraso da implementação das medidas necessárias é o fator determinante da fatalidade.

Por fim, os autores concluem que a adequada assistência hospitalar poderia ter contribuído para evitar uma proporção significativa dos óbitos maternos no Paraná, nos períodos estudados. Reiteram a necessidade de capacitação dos profissionais em todos os níveis de atenção à saúde e a necessidade de os gestores e profissionais dos hospitais analisarem os indicadores maternos e perinatais com a recomendação de estudos detalhados para cada hospital. De fato, Karolinski et al. ${ }^{16}$ observaram, em maternidades selecionadas de dois países sul-americanos (Argentina e Uruguai), que a utilização global de práticas baseadas em evidências na assistência materna era inferior a $60 \%$ na atenção de casos de morte materna e near miss. Os índices mais elevados da adoção de práticas baseadas em evidências foram nas emergências obstétricas, incluindo transfusões, cesárea oportuna e a administração de sulfato de magnésio em eclâmpsia. Em contrapartida, nas intervenções preventivas, como na administração de sulfato de magnésio na pré-eclampsia, ou o uso de antibióticos para rotura prematura das membranas, as taxas de utilização foram inferiores a $30 \%$.

No Brasil, já foram alcançadas as condições objetivas para avançar em resultados maternos e perinatais. Os marcos legais são suficientes e complexos, os recursos técnicos e as tecnologias estão presentes, e existe uma vigorosa formação de recursos humanos com ampla produção de pesquisa. Pode-se discutir em cada ponto as lacunas ainda existentes e o mergulho necessário em temas complexos, como o adequado uso de recursos financeiros, mas o ponto de inflexão necessário só virá quando, no individual e no coletivo, a morte materna de fato significar para cada um de nós a injustiça e a tragédia social que representa.

\section{Agradecimentos}

A Jose Guilherme Cecatti, por sua leitura e sugestões oportunas ao editorial. À Daíla Nina Ribeiro Freire, pela leitura e revisão ortográfica. À Alicia Fernandez, pela organização das referências bibliográficas.

\section{Referências}

1. World Health Organization. [Internet]. Trends in maternal mortality: 1990 to 2010. Geneva: WHO; 2010 [cited 2012 Oct 11]. Available from: http://whqlibdoc.who.int/ publications/2012/9789241503631_eng.pdf

2. Lozano R, Wang H, Foreman KJ, Rajaratnam JK, Naghavi M, Marcus JR, et al. Progress towards Millennium Development Goals 4 and 5 on maternal and child mortality: an updated systematic analysis. Lancet. $2011 ; 378(9797)$ : 1 139-65.
3. Organización Panamericana de la Salud. Organización Mundial de la Salud. Situación de salud en las Américas: indicadores básicos 2012[Internet]. Washington (DC): OPS; 2012 [citado 2012 Out 17]. Disponível em: http://argentina.campusvirtualsp. org/?q=node $/ 383$

4. United Nations Global strategy for women's and children's health [Internet]. New York: UN; 2010 [cited 2012 Oct 11]. Available 
from: http://www.everywomaneverychild.org/images/content/ files/global_strategy/full/20100914_gswch_en.pdf

5. United Nations. Millennium Development Goals: we can end poverty 2015[Internet]. New York: United Nations; 2010 [cited 2012 Apr 7]. Available from: http://www.un.org/millenniumgoals/bkgd.shtml

6. Centro Latinoamericano de Perinatología, Salud de la Mujer y Reproductiva. Plan de acción para acelerar la reducción de la mortalidad materna y la morbilidad materna grave: estrategia de monitoreo y evaluación. Montevideo: CLAP/SMR; 2012. (CLAP/ SMR. Publicación Científica; 1593).

7. Brasil. Ministério da Saúde. Portal da Saúde [Internet]. Rede Cegonha conta com quase $R \$ 9,4$ bi para garantia da assistência segura e humanizada à mãe e ao bebê: noticias. Brasília (DF): Ministério da Saúde; 2011 [citado 2012 Out 11]. Disponível em: http://portal.saude.gov.br/portal/aplicacoes/noticias/default. cfm?pg=dspDetalheNoticia\&id_area=124\&CO_NOTICIA= 12362

8. World Health Organization. The world health report: health systems financing: the path to universal coverage. Geneva: WHO; 2010.

9. Souza et al. Causas de mortalidade materna segundo níveis de complexidade hospitalar. Rev Bras Ginecol. 2012;34(12):536-43.

10. Soares VMN. Desigualdades na saúde reprodutiva das mulheres no Paraná. Rev Bras Epidemiol. 2007; 10(3):293-309.

11. Predebon KM, Mathias TAF, Aidar T, Rodrigues AL. Desigualdade sócio-espacial expressa por indicadores do Sistema de
Informações sobre Nascidos Vivos (SINASC). Cad Saúde Pública. 2010;26(8):1583-94.

12. Koch E, Thorp J, Bravo M, Gatica S, Romero CX, Aguilera H, et al. Women's education level, maternal health facilities, abortion legislation and maternal deaths: a natural experiment in Chile from 1957 to 2007. PLoS One. 2012;7(5):e36613.

13. Karlsen S, Say L, Souza JP, Hogue CJ, Calles DL, Gülmezoglu AM, et al. The relationship between maternal education and mortality among women giving birth in health care institutions: analysis of the cross sectional WHO Global Survey on Maternal and Perinatal Health. BMC Public Health. $2011 ; 11: 606$.

14. Nyamtema AS, Urassa DP, van Roosmalen J. Maternal health interventions in resource limited countries: a systematic review of packages, impacts and factors for change. BMC Pregnancy Childbirth. 2011;11:30

15. Rudge MV, Maestá I, Moura PM, Rudge CV, Morceli G, Costa RA, et al. The safe motherhood referral system to reduce cesarean sections and perinatal mortality - a cross-sectional study [19952006]. Reprod Health. $2011 ; 8: 34$.

16. Karolinski A, Mazzoni A, Belizán JM, Althabe F, Bergel E, Buekens P. Lost opportunities for effective management of obstetric conditions to reduce maternal mortality and severe maternal morbidity in Argentina and Uruguay. Int J Gynaecol Obstet. 2010;110(2):175-80. 